\title{
EDITORIAL
}

\section{The effectors of innate immunity: DAMPs, DAMEs, or DIMEs?}

\author{
David S Pisetsky ${ }^{1,2}$
}

'A rose by any other name would smell as sweet' is one of the most famous lines in all of literature. The implication of this observation, spoken by Juliet about the troubles in Verona because Romeo was called Montague and Juliet Capulet, is that names do not matter; rather, it is the thing itself that is important. Of course, Shakespeare's great line works because everyone knows what a rose is and that it smells sweet. What happens when the essence of a thing is not known and the name really matters?

I think that such a situation is occurring right now in the field of innate immunity since the essence of many things is not fully known. As research progresses rapidly, new cells, molecules, and pathways are discovered and receive names, often an acronym or an abbreviation or sometimes a brand new coinage. Dendritic cells, Toll receptor, and inflammasomes are all of recent origin. The derivation of such words is often fascinating. After all, 'toll' comes from the German word meaning 'great' and became famous when a researcher working on Drosophila excitedly exclaimed 'Das ist ja toll' ('That's great') when a critical immune defense system was discovered. To immunologists, toll now signifies a pattern recognition receptor (PRR).

One area in which nomenclature appears especially uncertain, ambiguous, and even controversial concerns the proper designation for molecules that are released from dead, damaged, and stressed cells to serve an immunological function in the extracellular space. These molecules have received various names, including danger molecules, alarmins, hyppos, and DAMPs. The current favorite seems to be DAMP, which stands for death (or danger or damage)-associated molecular pattern. An appealing feature of the word DAMP is the analogy with PAMP, or pathogen-associated molecular pattern.

Correspondence: dpiset@acpub.duke.edu

${ }^{1}$ Medical Research Service, Durham Veterans Medical Center, 151G Durham VA Medical Center, 508 Fulton Street, Durham, NC 27705, USA

2Department of Medicine, Duke University Medical Center, Durham, NC 27705, USA
PAMPs are foreign molecules that can stimulate PRRs such as the Toll-like receptors.

As a term, PAMP is ascendant although it may not be strictly correct since many of the molecules in question are present in organisms whether or not they are pathogens. All Gram-negative bacteria have lipopolysaccharide as well as C-phosphate-G DNA, but the content of these PAMPs does not determine pathogenicity. Despite quibbling about this probably minor point, scientists seem happy with the name and are unlikely to convene a consensus conference to come up with perhaps a more accurate descriptor.

As someone who likes the term alarmin, I have a certain reservation about the term DAMP. Certainly, damp is not a strong word. Damp is neither wet nor dry and conjures up unpleasant moisture that is noisome or nagging. Things that are damp are usually cool (a damp cloth on the forehead can soothe a fever), but a DAMP jettisoned from a damaged cell ignites a local conflagration, inducing the calor, rubor, tumor, and dolor that are the hallmarks of inflammation. Alarmin has a more decisive and compelling quality since an alarm is a clarion call to action, a sharp, often strident sound that gets adrenalin flowing and the heart pumping.

My other reservation about the term DAMP is that it does not fully capture the activity of these molecules or the structures in which these molecules reside. These structures include NETs (neutrophil extracellular traps produced during NETosis, another new word) and microparticles. NETs are literally net-like structures formed by the mixture of nuclear, cytoplasmic, and granular contents of neutrophils to form a mesh studded with antibacterial molecules. In contrast, microparticles are more discrete extracellular organelles that are a rich source of immunostimulatory molecules such as cytokines (for example, interleukin-1), HMGB1 (a classic alarmin), and histones; all of these molecules are potent immune stimulators.

While these molecules and structures can signal via PRRs as well as cytokine receptors, they have other 
important immune functions that extend well beyond activating cells during innate immune responses. These other functions do not result from interactions with PRRs. Thus, NETs are key elements in defense against organisms like bacteria and fungi, physically entrapping them so that they can be immobilized and killed (or at least slowed down) by the antibacterial components present. These components include histones as well as enzymes that can digest organisms as well as produce toxic reactive oxygen species to deliver a lethal hit. Like many other elements of the immune system, NETs can do harm and can damage endothelial and epithelial cells, using the same weapons that work against bacteria to injure host tissues to promote pathogenesis. NETs also promote thrombosis, which is a common event during infection and inflammation.

Among DAMPs, histones do not get the attention they deserve, but are overshadowed by HMGB1, another resident of the nucleus which can stimulate inflammation. HMGB1 has attracted great investigative interest because of its role as a key mediator of infectious and inflammatory diseases. Despite its high degree of conservation among species, HMGB1 has a quixotic and itinerant existence, circulating around the nucleus, touching but not embracing chromatin, migrating in and out of the cytoplasm depending on the state of the cell. When HMGB1 emerges from the cell during apoptosis, necrosis, or pyroptosis, it can have redox and other posttranslational modifications that determine its activity. In contrast, histones seem more fixed and stable, confined to the nucleus and tightly wrapped around DNA to form the nucleosome structure. Only during cell death do histones venture forth in the wide open spaces of the bloodstream, where they can kill bacteria and host cells alike as well as make the blood clot.

This is a quickly moving field as a host of interesting and provocative experiments indicate that DAMPs (and related structures) are more than just patterns to be recognized. They are effector molecules that are critical to host defense as well as the immunopathogenesis of conditions such as sepsis, lupus, and vasculitis. They are also targets for new therapies in diseases such as sepsis, in which reduction in histone levels by anti-histone antibodies or activated protein $\mathrm{C}$ (which degrades histones) is effective in shock models in animals. If the term DAMP was retained, the 'P' could stand for player and not pattern, but unless a comparable change was made for PAMP, confusion would follow.

These considerations suggest the need for a new term for DAMPs which highlights their functional activity and central role in thrombosis as well as immunity. The letter ' $E$ ' seems obvious for a new term. 'E' can stand for effector. 'E' can also indicate executioner (able to kill both bacteria and host cells) and, to the extent that some DAMPs carry information to distant sites, envoy. Alas, although E seems appropriate for inclusion in a new acronym, DAME (damage-associated molecular effector) may not make it. Political correctness could preclude the use of DAME from the start, although it has a ready-made slogan from the Broadway musical South Pacific. As the love-starved American Seabees sang about their fate on an island, 'There is nothing like a dame'.

Another possible term for at least some DAMPs is DIME, or death-induced molecular effector. Whereas the synthesis of these molecules is not induced by death, their translocation and exposure to the extracellular milieu are clearly induced by death and not just associated with cellular demise. Importantly, in an emerging conceptualization of their activity, DIMEs not only originate with death, they propagate it. Although the mechanisms are not certain, DIMEs (or DAMPs) likely contribute to rolling waves of death in situations such as chemotherapy, drug-induced liver injury, and chronic obstructive pulmonary disease, in which dying cells release their cytotoxic contents to damage and kill their neighbors.

While I think that the term DIME signifies another dimension of the activity of DAMPs, most of the expressions using the word dime have a negative connotation: 'dime store,' 'a dime a dozen', and, worst of all, 'not worth a dime'. Interestingly, the expression 'nickel and dimed to death' already exists and suggests some inkling about the danger of DIMEs.

At this point, although I enjoy thinking about nosology, I am more interested in the biology of the system and in the killing activity of molecules such as histones which can pop holes into cells and really can be deadly. Many questions arise. Are all histones killers? Do antihistone antibodies protect against cell injury in diseases such as lupus? Does citrullination affect the effector function of histones? Can the killing activity of histones be harnessed as a new type of antibiotic?

I am sure that someone can come up with a better name than DIMEs for these effectors and I would welcome alternatives. In the interim, I have put in my two cents and hope that more immunologists get off the dime and start to do more research on a fascinating group of molecules whose importance outside the cell may rival their importance inside.

Published: 31 Oct 2013

\section{Suggested reading}

1. Barrero CA, Perez-Leal O, Aksoy M, Moncada C, Ji R, Lopez Y, Mallilankaraman K, Madesh M, Criner GJ, Kelsen SG, Merali S: Histone 3.3 participates in a self-sustaining cascade of apoptosis that contributes to the progression of chronic obstructive pulmonary disease. Am J Respir Crit Care Med 2013, 188:673-683. 
2. Brinkmann $V$, Zychlinsky A: Neutrophil extracellular traps: is immunity the second function of chromatin? J Cell Biol 2012, 198:773-783.

3. Fuchs TA, Brill A, Duerschmied D, Schatzberg D, Monestier M, Myers DD Jr, Wrobleski SK, Wakefield TW, Hartwig JH, Wagner DD: Extracellular DNA traps promote thrombosis. Proc Natl Acad Sci U S A 2010, 107:15880-15885.

4. Harris HE, Andersson U, Pisetsky DS: HMGB1: a multifunctional alarmin driving autoimmune and inflammatory disease. Nat Rev Rheumatol 2012, 8:195-202.

5. Saffarzadeh M, Juenemann C, Queisser MA, Lochnit G, Barreto G, Galuska SP, Lohmeyer J, Preissner KT: Neutrophil extracellular traps directly induce epithelial and endothelial cell death: a predominant role of histones. Plos One 2012, 7:e32366.

6. Xu J, Zhang X, Pelayo R, Monestier M, Ammollo CT, Semeraro F, Taylor FB, Esmon NL, Lupu F, Esmon CT: Extracellular histones are major mediators of death in sepsis. Nat Med 2009, 15:1318-1321.

\subsection{6/ar4363}

Cite this article as: Pisetsky: The effectors of innate immunity: DAMPs, DAMEs, or DIMEs?. Arthritis Research \& Therapy 2013, 15:123 